\title{
EXPERT REVIEWS \\ Systems biology and modeling in neuroblastoma: practicalities and perspectives
}

Expert Rev. Mol. Diagn. 10(2), xxx-xxx (2010)

Jennifer A Logan, Martin E Kelly, Duncan Ayers, Nicholas Shipillis, Gerold Baier and Philip JR Day ${ }^{\dagger}$ ${ }^{\dagger}$ Author for correspondence Quantitative Molecular Medicine, Faculty of Medicine and Health Sciences, The Manchester Interdisciplinary Biocentre, University of Manchester, 131, Princess Street, Manchester, M1 7DN, UK

Tel.: +441612751621

Fax: +441612751617

philip.j.day@manchester.ac.uk

Neuroblastoma (NB) is a common pediatric malignancy characterized by clinical and biological heterogeneity. A host of prognostic markers are available, contributing to accurate risk stratification and appropriate treatment allocation. Unfortunately, outcome is still poor for many patients, indicating the need for a new approach with enhanced utilization of the available biological data. Systems biology is a holistic approach in which all components of a biological system carry equal importance. Systems biology uses mathematical modeling and simulation to investigate dynamic interactions between system components, as a means of explaining overall system behavior. Systems biology can benefit the biomedical sciences by providing a more complete understanding of human disease, enhancing the development of targeted therapeutics. Systems biology is largely contiguous with current approaches in NB, which already employ an integrative and pseudo-holistic approach to disease management. Systems modeling of NB offers an optimal method for continuing progression in this field, and conferring additional benefit to current risk stratification and management. Likewise, NB provides an opportunity for systems biology to prove its utility in the context of human disease, since the biology of NB is comprehensively characterized and, therefore, suited to modeling. The purpose of this review is to outline the benefits, challenges and fundamental workings of systems modeling in human disease, using a specific example of bottom-up modeling in NB. The intention is to demonstrate practical requirements to begin bridging the gap between biological research and applied mathematical approaches, for the mutual gain of both fields, and with additional benefits for clinical management.

KEYWORDs: biomarkers $\bullet$ modeling $\bullet$ MYCN $\bullet$ neuroblastoma $\bullet$ retinoic acid $\bullet$ systems biology

\section{Neuroblastoma}

Neuroblastoma (NB) is one of the most frequently occurring malignancies in childhood, accounting for $7-10 \%$ of all childhood cancers [1]. It is predominantly a malignancy of younger children, with the median age at diagnosis being 22 months and with $95 \%$ of cases diagnosed before the age of 10 years [2]. NB is an embryonal cancer that derives from neural crest cells, which are the primordial cells of the sympathetic nervous system [3]. Tumorigenesis from a neural crest cell involves failure of differentiation followed by uncontrolled proliferation [3]. The transforming genetic event is as yet unknown and may not be uniform across all cases [4]. Indeed, the array of genetic aberrations detected in NB tumors has led to a proposal that multiple genetic modulations interact in order to initiate and propagate tumorigenesis [1]

\section{Clinical presentation \& classification}

The clinical manifestations of NB are highly variable between individual patients [5]. Broadly speaking, presentation is with one of three clinical scenarios [1]: locoregional disease, metastatic disease or special metastatic disease. Locoregional disease presents with signs or symptoms of a space-occupying lesion in an otherwise healthy child, such as a palpable abdominal or pelvic mass or spinal nerve compression may produce neurological symptoms due to spinal nerve compression. Metastatic disease can have an identical presentation to locoregional disease but in addition the child may be generally unwell due to a high tumor burden, or may complain of pain following bony metastases. Special metastatic disease (MS or $4 \mathrm{~S}$ disease) presents in children younger than 18 months, typically with a small primary tumor and with metastases limited to 
the liver, skin and bone marrow. These cases are considered distinct from other metastatic NB because of their tendency to undergo spontaneous regression, hence their relatively good prognosis [6].

As hinted earlier, the degree of tumor invasion and metastasis at presentation is an important indicator of prognosis in NB. To assist risk stratification, various staging systems have been proposed that classify NB on the basis of tumor burden and spread. The most recent consensus system is the International Neuroblastoma Risk Group's (INRG) Staging System [7], summarized in Box 1. Unlike previous staging systems, which were based on surgical outcomes [8], the INRG system uses image-defined criteria (such as encasement of major vessels) and is, therefore, not biased by the timing or technique of operative procedures.

Shimada et al. demonstrated that histological analysis of biopsy material can also provide useful prognostic information in NB [9]. This study showed aggressive NB tumor behavior and poor prognosis are frequently associated with specific histological features, such as immature (undifferentiated) cell morphology and poorly developed Schwannian stroma. These initial observations led to development of the International Neuroblastoma Pathology Classification (INPC) [10], which remains in current use for the histologic and prognostic classification of individual NB tumors.

\section{Prognostic biomarkers \& risk stratification}

A variety of phenotypic and genotypic characteristics can be used to predict NB tumor behavior on an individual case basis. Noted previously, the tumor stage and histology are prognostically valuable, as is the patient's age at presentation [11]. In addition, the measurement of various molecular markers can assist in further evaluating the aggressiveness of the tumor and planning appropriate therapy [12]. Figure 1 provides an overview of biomarkers that have been characterized in NB, representing all levels of molecular organization. Historically, the clinical utility of these biomarkers has not always been clear and risk stratification has been limited to a restricted panel of the most robust biomarkers. Recently, a large cohort study by the INRG $(n=8800)$ has clarified the prognostic significance of many NB biomarkers, creating new advances in risk stratification [13]. Figure 1 includes the hazard ratios that were calculated from the results of the INRG study, indicating which of the listed biomarkers have the greatest predictive power.

Box 1. The International Neuroblastoma Risk Group Staging System.

- L1: Localized tumor not involving vital structures as defined by specified image-defined risk factors, and confined to one body compartment

- L2: Locoregional tumor with presence of one or more imagedefined risk factors

- M: Distant metastatic disease (except stage MS)

- MS: Metastatic disease in children under 18 months of age with metastases confined to skin, liver and/or bone marrow

Data from the International Neuroblastoma Risk Group Staging System [7].
As expected, the presence of metastatic disease (other than stage MS disease) and/or the presence of unfavorable histology were associated with an increased risk of events in the INRG cohort [13]. Using regression analyses, stage and histology were found to be the most statistically significant denominators for predicting survival. Beyond this, molecular markers were found to have a degree of prognostic value, with some more predictive than others. It is of note that no single molecular marker had greater prognostic value than stage and histology, and that individual biomarkers were typically only significant within phenotypic subgroups.

In the majority of phenotypic subgroups, amplification of the $M Y C N$ oncogene was associated with considerably worse survival compared with nonamplification [13]. This is in agreement with a large body of evidence indicating that $M C Y N$ amplification is a significant molecular biomarker for poor prognosis in NB [14-16]. Near-diploidy was also found to carry a poor prognosis, although only in patients younger than 18 months of age and with metastatic (non-MS) disease. Again, this corroborates the findings of previous work $[15,17,18]$. Nonrandom chromosomal abnormalities frequently occur in NB and have been proposed as prognostic indicators; for example, loss of heterozygosity at $11 \mathrm{q}$ or $1 \mathrm{p}$, and chromosomal gains at $17 \mathrm{q}$ are features quoted as typical of aggressive NB [19-21]. The INRG study demonstrated increased risk associated with these chromosomal abnormalities, although only $11 \mathrm{q}$ aberrations were found to have prognostic value within the regression analysis. The literature suggests various other genetic aberrations that may predict survival in NB $[22,23]$, but given the unavailability of data, these have not been analyzed in the INRG cohort. The current anticipation is that future developments of the INRG work will incorporate more extensive genetic data, as genome-wide technologies become more freely available [24].

Journals also contain multiple examples of putative NB biomarkers operating at the level of the transcriptome, proteome and metabolome $[12,25,26]$. Few of these could be analyzed in the INRG study, again due to unavailability of the relevant data. The serum biomarkers lactate dehydrogenase (LDH) and ferritin were analyzed and found to have prognostic significance within certain phenotypic and genetic subgroups; because of the small magnitude of their effects, these variables were not included in the final risk group classification.

The outcome of the INRG study was a new, more detailed risk -stratification system for NB, comprising 16 relatively homogeneous risk groups [13]. Risk stratification in NB is crucial to rational management decisions, determining which tumors require aggressive multimodal therapy and which are safe to manage conservatively. Therefore, risk stratification reduces morbidity and mortality, both from the disease itself and from agents used in its treatment [27].

\section{The need for a new approach in NB risk assessment}

Despite advances in risk stratification, subgroups of NB patients still have a dismal prognosis due to disease relapse and treatment resistance [1]. Targeted therapies for NB have thus far had limited 
success [28], possibly due to the heterogeneity of this disease. The aim of targeted therapy is to selectively modify a biological pathway that is unique to cancer cells, thus targeting the tumor but sparing normal cells. Unfortunately, NB does not appear to have a single crucial pathway that can be targeted, but rather permutations of multiple pathways at multiple levels of molecular organization (FIgURE 1). Modification of the biology of all NB tumors with a single targeted agent may not be possible.

We support arguments that to further improve the treatment of $\mathrm{NB}$ requires new approaches with enhanced utilization of biological data. These new approaches should consider NB biology holistically, rather than focusing on isolated biomarkers, which alone do not represent good targets for treatment. New strategies should also consider the functional and regulatory significance of the various biomarkers, since the mechanisms uncovered may be amenable to manipulation for therapeutic gain. Studying NB in this manner will provide a clearer understanding of the disease behavior, facilitating the rational design of safe drugs and effective therapies. One approach that could feasibly meet the above expectations is systems biology.

The aim of this review is to introduce the idea of systems biology and mathematical modeling in the context of NB. We explore whether modeling can offer additional benefits in conjunction with existing research approaches, with the potential to further advance clinical management.

\section{Potential of systems biology in the biomedical sciences}

Arguably, traditional biomedical research is focused primarily on individual genes, proteins or other singular markers, and therefore cannot adequately explain how these molecules interact to produce a functioning phenotype [29]. Systems biology emphasizes a more holistic approach, in which all components of a biological system carry equal importance [29]. Systems biology utilizes computer modeling and simulation to investigate the dynamic interactions between system components, as a means of explaining overall biosystem behavior [30]. The Holy Grail of systems biology is to achieve an understanding of biological systems in terms of control and function of their dynamic entirety (i.e., whole living organisms).

Kitano claims that for systems-level understanding, insight is required into four key properties: system structure (individual components and interactions between them), system dynamics (system behavior under different conditions and over time), control methods (principles governing regulation of the system), and design methods (principles governing system construction) the INRG study).
Prognostic indicator

MYCN amplification

Near diploidy (versus hyperploidy)

Chromosome $1 p$ aberration

Chromosome 11q aberration

Chromosome $17 q$ gain

ALK amplification and/or mutations

Increased expression of MYCN, ALK,

trkB, telomerase

Decreased expression of trkA, trkC, CD44

Increased expression of $\mathrm{N}$-myc, ALK, trkB, telomerase

Decreased expression of trkA, trkC, CD44

Increased serum lactate

dehydrogenase (>587 U/I)

Increased serum ferritin (>92 ng/ml)

Increased serum neuron-specific enolase

Unfavorable INPC histology classification

Metastatsis to distant organs

(excluding MS disease)
Hazard ratio

4.1

2.3

3.2

2.3

1.7

$-$
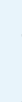

$-$

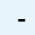

2.4

3.6

6.6

5.2
Figure 1. Overview of biomarkers with proven or potential prognostic value in neuroblastoma. A range of phenotypic characteristics and molecular markers have been proposed to have value in predicting outcome in NB. The quoted INRG hazard ratios denote increased risk of an event in the presence of a biomarker, compared with the absence of that biomarker; null values indicate biomarkers that were not analyzed by

[31]. This approach makes possible the full understanding of the organism's phenotype and the intelligent manipulation of the system for desired phenotypic effects. Alternative manifestos for systems biology differ in their precise details but the principles and anticipated benefits are the same [32,33].

The pharmaceutical industry has begun to take an active interest in systems biology to gain from the potential benefits offered in improving and hastening the drug-development process. Currently, preclinical drug development involves a lengthy process of in vitro and animal testing, which may be rendered of no therapeutic use if the drug shows toxic or other unexpected effects in humans. A dynamic molecule-to-phenotype model of the human system would allow in silico drug screening, and, thus, more efficient and ethical use of time and resources. It might also be possible to reverseengineer compounds based on the desired system effects, avoiding unwanted toxicity and allowing drug design rather than discovery. Although a systems-level approach to drug design is still very much in its infancy, there are many leading authorities who recognize the potential of systems biology in clinical therapeutics [34-37].

Systems biology is also of interest in the field of personalized medicine [38]. In this context, the aim of systems biology is to identify and model key system components (e.g., genes and proteins) that regulate a phenotypic behavior of interest. By measuring these key molecules, the model can be tailored to represent the biology of an individual patient. The personalized model could 
Box 2. Main characteristics of top-down and bottom-up systems biology.

Top-down systems biology

- From big picture down to fine detail

- Inductive approach

- Starts with global system data ('omics)

- Uses computer modeling and statistics

- Proposes interactions/mechanisms to explain global data (hypotheses)

- Performs experiments to test hypotheses

- Iterative cycle leads to a better model each time

- Advantages:

- Comprehensive (potentially complete) datasets

- Focuses on system-level behavior and phenotype

- Disadvantages:

- Models not grounded in biological knowledge

- Statistics may not accurately reflect biology

\section{Bottom-up systems biology}

- From fine detail up to big picture

- Deductive approach

- Starts with local mechanistic data (e.g., kinetics)

- Uses formulation and integration

- Generates a mechanistic model of the subsystem

- Combines subsystem models into a global model (modular approach)

- Accumulation leads to bigger model each time

- Advantages:

- Models grounded in biological knowledge

- Parameters are determined experimentally

- Disadvantages:

- Difficult to obtain accurate mechanistic data

- Combining models leads to cumulative errors

be used to identify signature molecules for screening or early diagnosis in phenotypically healthy patients, or for predicting prognosis at any stage of the disease. A systems approach could also be used to predict the response to the available treatments and indicate the best therapy on an individual basis [39].

\section{Top-down \& bottom-up systems biology}

Systems biologists typically take either a top-down or a bottom-up approach to the study of biological systems [29]. The top-down approach starts with global systems-level data and aims to characterize the underlying regulatory mechanisms. This approach has been facilitated by the increasing availability of high-throughput 'omics technologies [40,41] and by continually improving modeling techniques [42-44]. The bottom-up approach starts with a mechanism-based model of a portion of the system (a subsystem) and integrates this with adjacent subsystems, building up to a whole system model. Here, the dominant requirement is for appropriate experimental design alongside quantitative measurement techniques, permitting the level of accuracy and detail needed for kinetic modeling [45]. Box 2 summarizes the important differences between the top-down and bottom-up approaches.
From a biomedical perspective, the ultimate goal for systems biology is to accurately model human health and disease at the molecular level, enabling effective drug design and a personalized approach to medicine. Currently, neither the top-down nor bottom-up approach is able to achieve this: the top-down approach lacks mechanistic detail; the bottom-up approach lacks breadth. As experimental and modeling techniques improve, both approaches will advance towards the ultimate goal, albeit from different angles. The prediction is that eventually the two approaches will converge into a single coherent strategy for the investigation and improvement of human health.

Even from their current polarized positions, both top-down and bottom-up systems biology can make complementary contributions to biomedical research. The top-down approach can identify profiles of biological activity that contribute to pathophysiology, enhancing understanding of disease etiology. Top-down work is also well suited to the discovery of diagnostic and prognostic biomarkers, which can be developed for use in the clinical setting [46]. By contrast, the bottom-up approach allows detailed modeling of the mechanistic and quantitative details of disease pathways. This can be used, for example, in the identification of novel targets for therapy or in predicting therapeutic response on an individual basis. Developments in both top-down and bottom-up systems biology can, therefore, generate novel approaches to the diagnosis and management of disease, which are directly transferrable to the patient's bedside.

\section{A systems approach to NB Benefits of systems biology for NB research}

Systems biology has much to offer NB, which is a complex disease still fraught with challenges and mysteries. The holistic and dynamic nature of systems biology could provide solutions to some of the current challenges in NB. For example, bottom-up work can clarify the mechanistic relationships underpinning biomarker associations, revealing biological networks that contribute to aggressive disease. By modeling these networks it is possible to identify which molecules influence the phenotypic behavior most directly, and are, therefore, suitable targets for the design of novel therapies. Top-down modeling may be of particular use in refining the current risk-stratification systems for NB: 'omics technologies provide an unbiased approach to biomarker discovery, and top-down modeling can then be used to identify relevant associations within the data. This approach could be used to create a detailed NB classification system that groups patients according to biological and not statistical likeness; this would allow an increasingly personalized management approach, with patients offered treatment that is most beneficial to their biological profile.

The principles of systems biology are in many ways a good fit with the current approaches to NB. Leading NB authorities have long realized the value of integrating clinical and biological data, and this strategy bears resemblance to the holistic and multiscale nature of a systems-biology approach. For example, NB risk stratification was initially based on gross phenotype observations (patient age and extent of tumor invasion), and progressed to 
incorporate evidence from the fields of histopathology [10] and molecular biology [12]. These developments have permitted the evolution of increasingly detailed and more accurate stratification systems, such as the INRG [13], accompanied by parallel advances in biological treatments for NB [47,48]. Systems biology is therefore contiguous with traditional NB research, and represents a means to enhance progression in this field. A systems-biology model of NB would add value to the existing work by aiding mechanistic understanding of the known clinical and biological associations.

\section{Benefits of NB research for systems biology}

From a systems biology perspective, NB may be an ideal human disease to model owing to the degree of molecular detail in which it has been characterized. NB biology has been studied using a range of quantitative techniques, covering many levels of molecular organization and diverse aspects of phenotypic behavior (FIGURE 1). These data could form the foundation for construction and validation of various models. Owing to its suitability for modeling, NB provides an excellent opportunity for systems biology to prove its utility in the study of human diseases. If systems biology can be successfully applied to NB and used to derive clinical benefits, this will justify the claims made by systems biology advocates and encourage others to take up this approach.

\section{Wider benefits of a systems approach to $N B$}

The successful construction of a NB disease model could facilitate the modeling of other embryonal cancers, such as Wilm's tumor and retinoblastoma, which share some of the biological characteristics of NB [49]. In addition, specific components of the NB model would have relevance to broader biological themes, such as growth factor signaling in embryonal development [3], and myc protein activity in oncogenesis [50]. In effect, the union of systems biology with NB has the potential to generate benefits not only for both of these fields but also for the wider biological community.

\section{Modeling \& simulation in systems biology}

Modeling and simulation are key components in the practical application of systems biology. They involve the use of mathematical means to replicate and manipulate biological systems in a virtual framework, leading to better understanding of how these systems function in reality. Modeling and simulation can offer novel benefits to the biomedical sciences that are complementary to the benefits of traditional experimental methods.
Modeling involves studying and reverse engineering biological networks, which can be defined as groups of molecules (nodes) connected by measurable interactions (edges). The mathematical description of such networks can reveal relationships that would be difficult to observe with traditional methods of data representation and analysis, which typically provide rather limited and static information regarding the biological relationships involved.

Simulation involves the in silico manipulation of various nodes within the mathematical model, allowing observation of the network response to a specific perturbation. Analyzing the results of simulations reveals dynamic interactions between system components that were not evident from the raw data [44,51]. In addition, the virtual nature of simulations allows for predictions and hypotheses that are unconstrained by the usual boundaries of wet-laboratory experiments.

Modeling and simulation can offer benefits in the study and management of any cancer or complex disease [52], and particularly for heterogeneous conditions, such as NB. For example, the
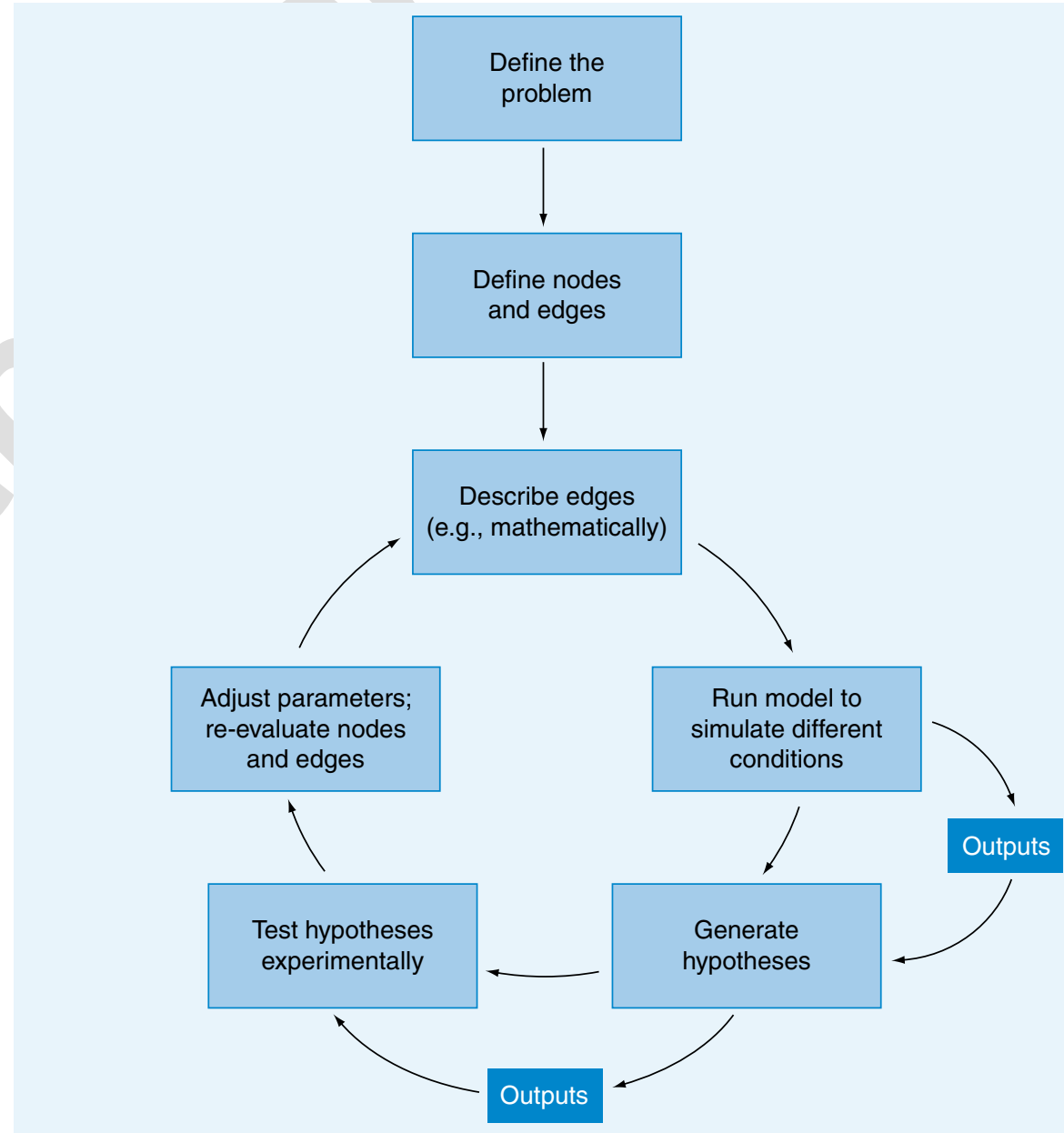

Figure 2. The general workflow used for model construction and validation. This iterative process requires alternating rounds of dry modeling and experimental validation. Useful outputs are yielded both from the simulations and the lab-based experimentation, issuing new information that guides the subsequent work and contributing to an enhanced understanding of the original problem. 
process of modeling characterizes the underlying molecular networks that contribute to the etiology and progression of disease, and may indicate potential targets for therapy. Simulation then allows potential therapeutic targets to be 'tested' in silico by simulating a perturbation and observing the effects on the network as a whole. Modeling and simulation can thus be used to design therapies based on the optimum predicted network response, taking into account all relevant interactions rather than focusing on an isolated pathway.

\section{A workflow for systems modeling: illustrated for retinoid response in NB}

Systems modeling and simulation can take diverse forms depending on the bioprocess examined and the data available; however, there is a generalized workflow that is followed in most instances, as shown in Figure 2. This section illustrates the execution of each stage in Figure 2, using a specific example of bottom-up modeling in NB.

\section{Defining the problem}

As with all research, the first stage of the modeling process is to clearly define the scenario or problem of interest. This is of fundamental importance since it largely determines the components of the model and the type of modeling approach used. The example here is concerned with the influence of 13-cis-retinoic acid on the $M Y C N$ regulatory network. The model is then used to simulate the response of an individual tumor cell to retinoid treatment.

Retinoids are employed as part of the extensive treatment regime for high-risk NB [53]. Following intensive cytotoxic treatment and surgery, 13-cis-retinoic acid is used for a period of 22 weeks to target minimal residual disease (MRD). The desired phenotypic response following administration of retinoids is cytostasis and cell differentiation, thus reducing the malignancy of residual tumor cells. Unfortunately, the response to retinoids is somewhat variable, with the result that some patients may endure unpleasant side effects of the drug for little if any therapeutic gain [54,55].

MYCN amplification occurs in approximately $22 \%$ of all NB tumors [56] and is associated with a dismal 34\% 5-year survival rate [13]. Consequently, almost all tumors with $M Y C N$ amplification are classified as high risk [13], and treated with cytotoxic and retinoid therapy, as described earlier. The $M Y C N$ protein product, $\mathrm{N}-\mathrm{Myc}$, functions as a transcription factor [57], binding and potentially regulating up to $10 \%$ of the genome [58]. In the context of $M Y C N$ amplification, N-Myc stimulates a diverse range of cell behaviors, including increased proliferation, loss of differentiation, drug resistance, angiogenesis, and genomic instability; $M Y C N$ amplification, therefore, contributes to tumorigenesis and malignant tumor behavior via multiple mechanisms [57].

From the literature, it appears that retinoids interact with the $M Y C N$ network and may exert their actions predominantly via these pathways. For example, $M Y C N$ stimulates progression through the cell cycle and prevents differentiation, by regulating the expression of many cell cycle proteins [59-66]. Retinoids produce the phenotypic responses of cytostasis and differentiation by directly or indirectly perturbing some of the same cell cycle proteins [67-70]. Furthermore, NB tumors that respond 
phenotypically to 13 -cis-retinoic acid show a decrease in $M Y C N$ expression, whereas those that do not respond display no decrease in $M Y C N$ expression [71]. Figure 3 depicts the biological interactions of $M Y C N$ and N-Myc that are relevant to the cell cycle, and indicates how retinoid perturbation influences these interactions.

\section{Selecting the nodes for the model network}

Once the context and purpose of the model have been defined, the next stage is to select relevant nodes that should be included in the model network. Network nodes may be genes, proteins, small molecules or clusters, depending on what type of network is most appropriate for addressing the biological problem. Nodes are initially identified and defined using information from the literature and from large-scale omics datasets, and there are a growing number of text-mining and bioinformatics tools available to enhance this process. The National Centre for Text Mining offers a range of text mining tools to allow automated extraction of relevant information from large collections of literature [201].

In the example model, the nodes are based on the biological $M Y C N$ regulatory network shown in Figure 3. Two of the fundamental nodes are MYCN mRNA and N-Myc protein concentrations. Additional nodes represent molecules that regulate $M Y C N$ expression and/or are modulated by N-Myc activity. To reduce complexity for the purpose of modeling, molecules with the same functional role have been condensed into a single node. The model, therefore, has six nodes, which are shown in Figure 4.

\section{Describing the edges of the model network}

Following selection of the relevant nodes, the model is completed by identifying and describing the edges between nodes. Relationships between nodes can be described in various ways, depending on the biological problem being modeled. In a Boolean model, edges are described using logic functions, which produce a qualitative description of the relationships between nodes. Boolean models are commonly used as a method for representing gene regulatory networks (GRNs) [44]. In a deterministic or stoichiometric model, edges are described mathematically using ordinary differential equations (ODEs), which allow for quantitative description of the relationships between nodes [42]. Stoichiometric models assume the context of a controlled experiment and, therefore, the model parameters are fixed at predetermined values derived from quantitative experimental data. In a stochastic model, edges are again described mathematically, but the model parameters are obtained from probability distributions, rather than being fixed. Stochastic models, therefore, incorporate an element of randomness, which in some instances may be a more appropriate representation of certain aspects of biological systems [44].

In the example model, the edges are defined as shown in Figure 5. The concentration of each node is determined by a rate of production and a rate of destruction, and is also modulated by the concentrations of other nodes, as indicated by the edges. Retinoids exert their effects by altering the rates of production of the CDKs/cyclins and inhibitors nodes. These relationships and dependencies have been described mathematically using ODEs, since the nature of the clinical problem necessitates a quantitative

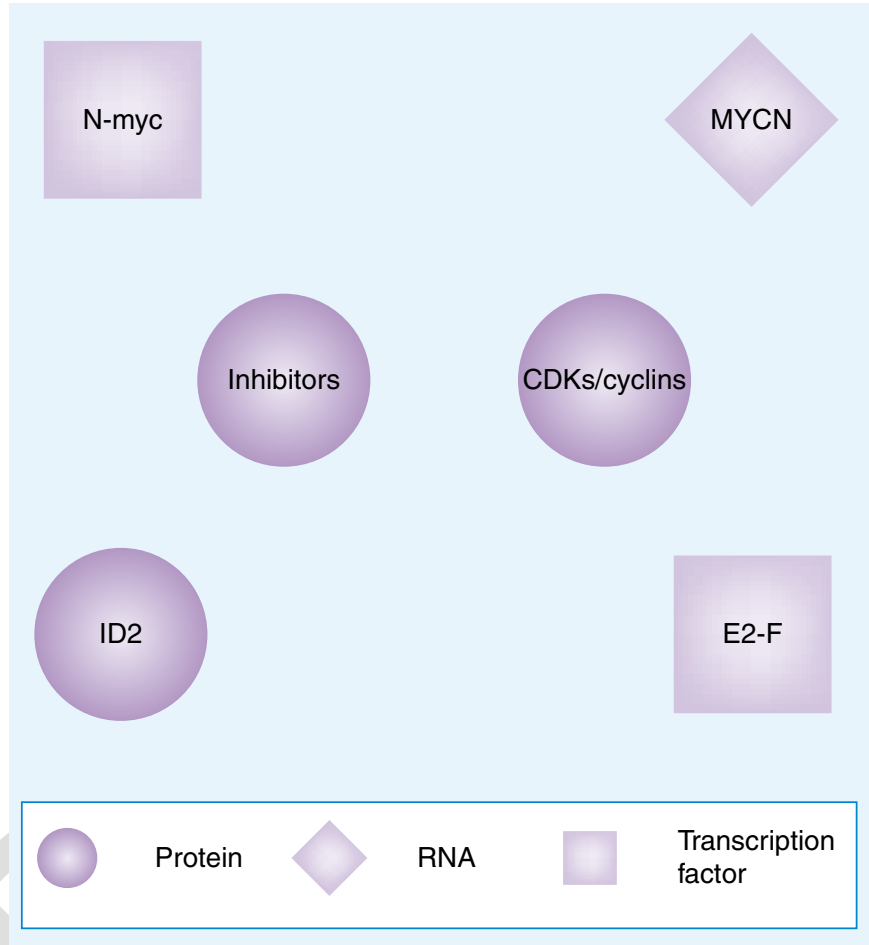

Figure 4. Defining nodes for inclusion in the 'silicon' network. The biological MYCN network has been simplified to allow for feasible modeling. The 'inhibitors' node represents the inhibitory cell cycle proteins p21 and p27. The 'CDKs/cyclins' node represents the CDK, cyclin and cdc25 proteins. Remaining nodes correspond directly to the biological network in Figure 3.

approach. However, in order to illustrate how ODEs have been formulated from the network structure, TABLE 1 provides a qualitative summary regarding the regulation of each node in the network. The equations shown in TaвLE 2 are subsequently a mathematical representation of these qualitative relationships.

\section{Utilizing the model to perform simulations}

When a mathematical model is run, the logic functions or differential equations are solved, producing output values for the model variables. If the model uses differential equations, these are integrated numerically, giving the concentration of each variable as it changes over time. Simulation is the process of scanning selected model parameters (varying parameter values over a range) and then integrating the model to examine the consequences on each of the involved variables and on the system as a whole. Simulations can be considered the computing equivalent of experiments, since they allow the researcher to generate and test hypotheses.

In addition to using fixed parameter values (mimicking a stable environment) simulations may represent intrinsic changes within the system, such as perturbations that alter the concentration or activity of a particular species [72]. Alternatively, they may seek to mimic the effects of external stimuli; for example, modeling the putative mode of drug action [51]. It should be noted that a number of user-friendly packages, such as COPASI [202] and BPS [203], are available to assist with the simulation and analysis of pathways/networks. 


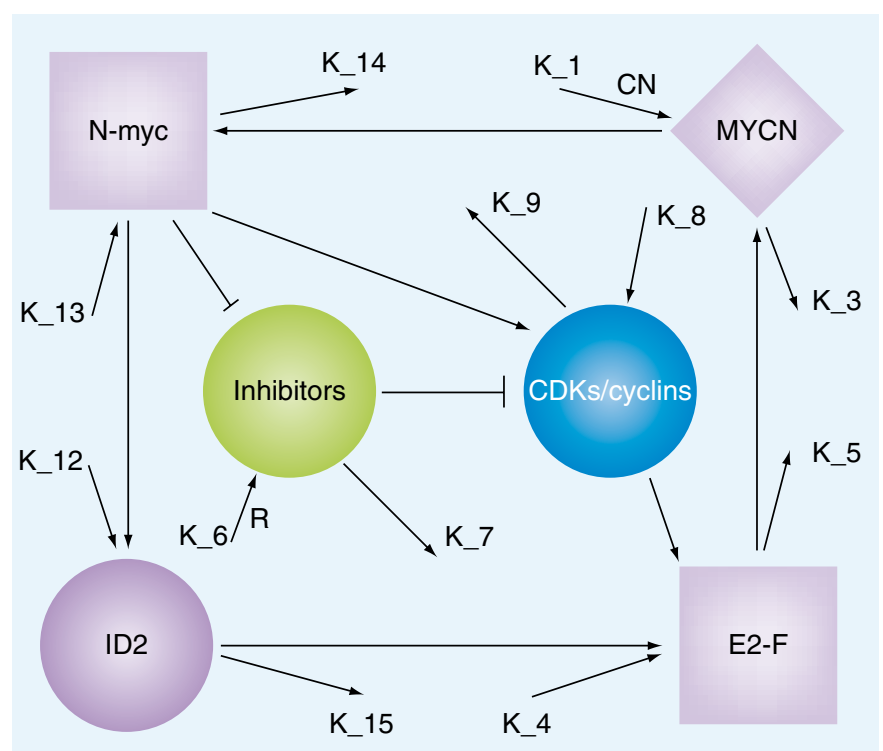

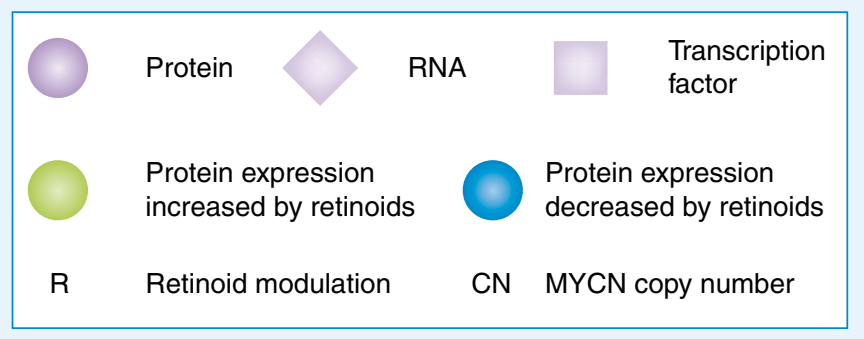

Figure 5. Defining the edges in the 'silicon' network. The concentration of each node is determined by a rate of production and a rate of destruction, illustrated by a value $K_{-} \#$ (constant). The rate of production of each node is dynamically regulated by the concentrations of other nodes. Retinoids perturb the silicon network through modulation of the 'inhibitors' and 'CDKs/cyclins' nodes, as in the biological network. TABLE 1 describes qualitatively the relationships between nodes. TaвLE 2 shows the differential equations that describe mathematically these dynamic relationships.

The above model has been used to simulate retinoid perturbation of the $M Y C N$ regulatory network. The $M Y C N$ copy number parameter was scanned from 0 to 200. For each copy number value, the model was allowed to run until all variables (nodes) reached steady state concentration. Retinoid activity was either present or absent. The output was a plot of $M Y C N$ expression steady state against $M Y C N$ copy number.

Figure 6 shows the simulation results of a 13-cis-retinoic acid perturbation, in comparison with the unperturbed system. The model assumes a hyperbolic relationship between $M Y C N$ copy number and $M Y C N$ expression. In reality, such a simplistic relationship does not exist, since the expression of $M Y C N$ is regulated by more molecules (nodes) than have been included in this model. Numerous studies have indicated that $M Y C N$ copy number is not independently predictive of $M Y C N$ expression and that $M Y C N$ overexpression in the absence of amplification has variable phenotypic effects [59,73-75]. We do not attempt to explain the MYCN paradox with this model as a far more complex model would be required. For the sake of simplicity, the model assumes $M Y C N$ copy number and expression are closely linked, and that a decrease in $M Y C N$ expression will have the phenotypic effect of cytostasis and differentiation, (i.e., a response to 13-cis-retinoic acid). The simulation results indicate that at a lower $M Y C N$ expression/copy number the system responds to 13-cis-retinoic acid, and MYCN expression decreases. However, above a certain $M Y C N$ expression threshold (arbitrarily at 2900 in this example), the system does not respond to 13-cis-retinoic acid.

The above simulation results give rise to the hypothesis that: tumor response to retinoids is dependent upon $M Y C N$ copy number and/or expression. From the literature, there is some evidence that NB tumors that initially express very high levels of $M Y C N$ respond poorly to 13-cis-retinoic acid [71]. This suggests that very high $M Y C N$ expression can create a state of system robustness against the effects of 13-cis-retinoic acid, which appears to be in keeping with the results of the simulation. Interestingly, these drug-resistant tumors respond to the synthetic retinoid fenretinide, indicating an alternative mechanism of action not encompassed in the above model [71]. The hypothesis generated could potentially explain why clinical response to retinoids is so variable, although experimental work would be required to validate these suggestions.

The potential clinical value of the above model is in predicting which NB tumors are likely to respond to 13-cis-retinoic acid, based on simultaneous pretreatment measurement of all relevant molecules. Ideally, only tumors that are expected to respond to 13-cis-retinoic acid should be treated with this drug; in tumors that are not expected to respond, the risk of toxicity is unacceptable given the improbability of benefit. If validated with experimental data, the model could potentially be used to alter the current management of high-risk NB: tumors with $M Y C N$ expression indicative of a good response to 13-cis-retinoic acid should be treated with this drug; tumors with higher $M Y C N$ expression should not receive 13-cis-retinoic acid, but may derive benefit from the alternative fenretinide (if licensed) [71].

\section{Experimental validation \& model optimization}

As shown in the previous section, simulations generate hypotheses regarding the properties or behavior of the biological system. Appropriate experimental work is then required to test these hypotheses, and determine whether or not the model provides an accurate representation of the biology. If experimental results differ from simulated predictions, the model is adjusted accordingly. Over iterative cycles of simulation and hypothesis testing, the model is continually improved to achieve a better representation of the biology (Figure 2) [44,76].

In addition to hypothesis testing, experimental work is also used for optimization of model parameters. Algorithms are used to fit the model to the experimental data, in order to determine the optimum value for each of the parameters [77,78]. Fitting to data is again an iterative process, with the model becoming increasingly accurate as the quality of experimental datasets improves.

Experimental work is needed to validate the above model and simulation results. Experiments should be conducted to determine whether system response to retinoids is indeed influenced by $M Y C N$ expression. 


\begin{tabular}{|c|c|c|c|c|}
\hline $\begin{array}{l}\text { Node/ } \\
\text { variable }\end{array}$ & $\begin{array}{l}\text { Rate of } \\
\text { production }\end{array}$ & $\begin{array}{l}\text { Production } \\
\text { regulated by }\end{array}$ & $\begin{array}{l}\text { Effect on } \\
\text { production }\end{array}$ & $\begin{array}{l}\text { Rate of } \\
\text { destruction }\end{array}$ \\
\hline MYCN & K_1 & $\begin{array}{l}\text { MYCN CN } \\
\text { E2-F }\end{array}$ & $\begin{array}{l}\text { Increased } \\
\text { Increased }\end{array}$ & K_3 \\
\hline E2-F & K_4 & $\begin{array}{l}\text { CDKs/cyclins } \\
\text { ID2 }\end{array}$ & $\begin{array}{l}\text { Increased } \\
\text { Increased }\end{array}$ & K_5 \\
\hline Inhibitors & K_6 & N-Myc & Decreased & K_7 \\
\hline CDKs/cyclins & K_8 & $\begin{array}{l}\text { N-Myc } \\
\text { Inhibitors }\end{array}$ & $\begin{array}{l}\text { Increased } \\
\text { Decreased }\end{array}$ & K_9 \\
\hline ID2 & K_12 & N-Myc & Increased & K_15 \\
\hline N-Myc & K_13 & MYCN & Increased & K_14 \\
\hline
\end{tabular}

Furthermore, the model should be fitted to quantitative experimental data, in order to optimize model parameters and estimate the $M Y C N$ expression threshold above which the system will not respond to 13-cisretinoic acid. If the model were experimentally validated and optimized, its potential clinical value is in predicting which tumors will respond to 13-cis-retinoic acid and which will not respond, based on pretreatment measurement of all relevant molecules.

\section{Considerations for human systems biology}

The purpose of this review is to emphasize the benefits that systems biology and mathematical modeling can bring to the study of human health, specifically the pediatric cancer, NB. The review also seeks to show 'how' models can be constructed and optimized to better represent disease processes. However, it is worth considering some of the more difficult and demanding elements of this approach, and the various initiatives being made to address them.

\section{Challenges of modeling the human system}

Systems biology has demonstrated preliminary successes within simple microbial and yeast biosystems [79-82], but modeling of higher organisms, including the human system presents further challenges. The principal problem is that current analytical and simulation techniques cannot cope with the biological complexity and diversity of the human organism.

Human biological complexity, in comparison with microbes and yeast, can be partially attributed to multicellularity. The human organism comprises various cell populations, which during development establish spatial organization in the form of tissues and organs [83]. In some respects, each individual cell functions as a self-contained biological system, with many molecular interactions and autoregulatory mechanisms $[84,85]$. The function of each cell system is also influenced by nearby cells which communicate via the cell microenvironment, often in the form of cell-cell interactions or paracrine signaling [86,87]. Furthermore, cell behavior can be modified by the activities of distant organs, either by specific neural connections [88], or by exo-metabolites and hormones secreted into biological fluids and distributed systemically [89]. This hierarchical regulation creates infinitely more possibilities for phenotypic behavior and, therefore, modeling the system accurately requires attention to both $3 \mathrm{D}$ structure [90] and compartmental separations [91].

Another contributory factor towards human complexity is the considerable variation between individuals, both inherited and acquired. The investigation of human genetic variation has been enabled by various new technologies; for example, allowing comprehensive genome sequencing [92] and population haplotyping [93]. Transcriptomics technologies add a functional dimension by providing holistic profiles of gene expression under different conditions $[94,95]$. The challenge for systems biology is to use appropriate top-down modeling techniques to extract relevant information from these large datasets, identifying the genetic variations that are biologically relevant. Biological variation between individuals is further compounded by environmental influences [96], which in the case of humans includes not only physical variables, such as nutrients [97], but also cultural, social and educational experiences [98-101], which arguably make the human system unique, even compared with its evolutionary relatives. Therefore to accurately model and understand human biology requires some consideration to the environmental context of the individual. Model organisms for human biological and disease processes may have a more limited role than which we have become accustomed to.

To compound these challenges further, there are important ethical issues and practical constraints when studying the human system. Genes cannot be knocked-down, growth conditions cannot be manipulated and many variables in the individual's environment cannot be controlled. Consequently, modeling the human system presents a demanding project, which may be best addressed with collaborative efforts.
Table 2. Mathematical description of the dynamic factors influencing node concentration.

Node/ Differential equation

variable

MYCN

$\mathrm{dMYCN} / \mathrm{dt}=0.00001+\mathrm{K}_{-} 1$ * $(1+\mathrm{E} 2 \mathrm{~F} \wedge \mathrm{p}) /\left(\mathrm{K}_{-} 11 \wedge \mathrm{p}+0.01\right.$ * E2F^p) $)$ * $(\mathrm{cn} \wedge \mathrm{m}) /\left(\mathrm{Km} \_1 \wedge \mathrm{m}+0.1{ }^{*} \mathrm{cn} \wedge \mathrm{m}\right)-\mathrm{K}_{-} 3 * \mathrm{MYCN}$;

E2-F

Inhibitors

$\mathrm{dE} 2 \mathrm{~F} / \mathrm{dt}=\mathrm{K} \_4$ * $(1+\mathrm{CDKsCyclins}) *(1+\mathrm{ID} 2)-\mathrm{K}_{-} 5$ * E2F

CDKs/Cyclins

dlnhibitors $/ d t=R$ * K_6/(Km_6 + NMyc^n) $-K_{-} 7$ * inhibitors

ID2

$\mathrm{N}-\mathrm{Myc}$
$\mathrm{dCDKscyclins} / \mathrm{dt}=\mathrm{K} \_8$ * NMyc/(Km_8 $+\mathrm{R}+\mathrm{NMyc}+$ inhibitors $\left.^{\wedge} \mathrm{O}\right)-\mathrm{K} \_9$

* CDKscyclins

$\mathrm{dID} 2 / \mathrm{dt}=\left(\mathrm{K} \_12 * \mathrm{NMyc} /\left(\mathrm{K} \_12 \mathrm{a}+0.01 * \mathrm{NMyc}\right)\right)-\mathrm{K} \_15$ * ID2

$\mathrm{dNMyc} / \mathrm{dt}=\mathrm{K} \_13$ * MYCN $-\left(\mathrm{K}_{-} 14\right.$ * NMyc) 


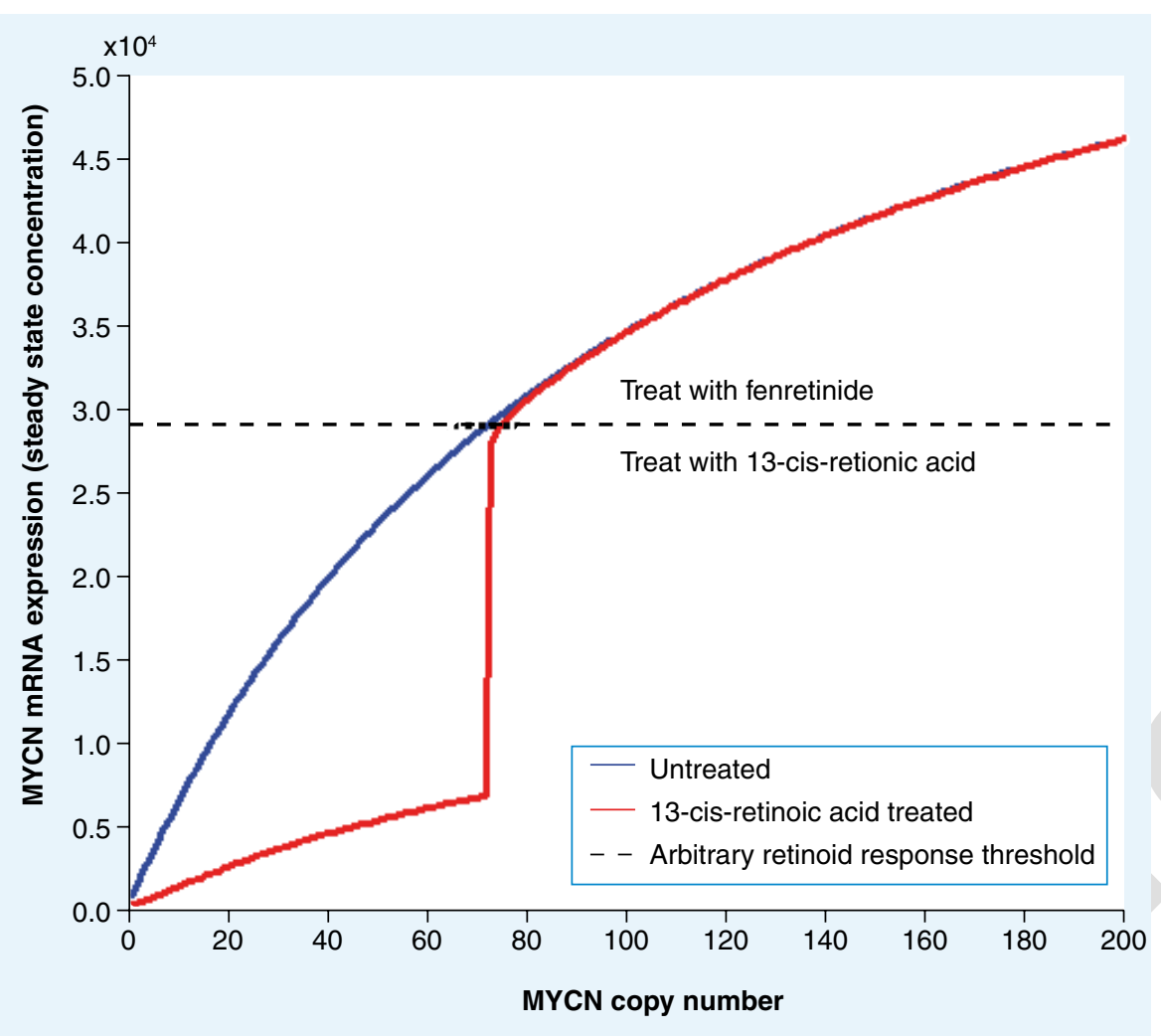

Figure 6. Simulation of a 13-cis-retinoic acid perturbation. The blue line shows MYCN mRNA expression in the absence of retinoid. The red line shows MYCN mRNA expression following perturbation with 13-cis-retinoic acid. Below the arbitrary retinoid response threshold, 13-cis-retinoic acid is able to decrease MYCN expression, indicating that the tumor should show a phenotypic response. Above this threshold, the system is robust to perturbation, indicating that the tumor will not respond and that an alternative therapy is required.

\section{Collaborative efforts towards human systems biology:} the need for standards \& frameworks

With the techniques currently available, considerable advances can be made in human systems biology, particularly when data and techniques are shared within the community. For this to be efficient, a degree of infrastructure is required.

Different 'omics data can make important contributions to human systems biology. However, in order for these data to benefit the wider community it must be of good quality and properly annotated. Numerous standards for data quality and annotation are emerging for different kinds of datasets, for example the Minimum Information About a Microarray Experiment (MIAME) [102] and Minimum Information for publication of Quantitative real-time PCR Experiments (MIQE) [103]. These standards aim to ensure that biological data made available to the community are accurate, valid and presented in its appropriate context.

Standards have also emerged to facilitate model compatibility. Previously, the various software packages used for modeling all used different formats and syntaxes, meaning that models created in different software environments could not be easily combined or compared. The development of Systems Biology Markup Language (SBML) has revolutionized model compatibility by providing a common intermediate format that can be used for representing and analyzing models, regardless of the programming language and software encoding used [104]. SBML significantly extends the usefulness of a given model beyond those with the same software, effectively to the whole community. SBML is integral to large collaborative modeling projects, such as the recent consensus reconstruction of the yeast metabolic network [80].

The modeling of human biology has also been the focus of collaborative efforts, for example, the IUPS Physiome Project [105]. This project has developed a framework allowing the connection and interaction of different models representing all aspects of human physiology. The infrastructure takes into account spatial and temporal organization of the human organism, and aims to represent all levels of biological function, from the behavior of organ systems down to molecular events at the cellular level. Once completed, the 'digital human' can be used as a basis for investigating the physiological effects of various perturbations, including individual genetic variation, environmental agents, disease processes and aging.

\section{Conclusions \& future directions}

A systems biology approach to human disease is challenging, but collaborative efforts are facilitating progression towards this ultimate goal. Rapid advances are being made in analytical technologies, bioinformatics and modeling, resulting in more accurate representation of biology with each iteration of the model development cycle. The sharing of data and techniques within the community facilitates progression in systems biology and is supported by standards for data quality and development of a common model format.

Although modeling techniques are far from offering a completely accurate representation of human disease, systems biology already has much to offer the fields of medicine and pharmaceutical design. It is particularly relevant in heterogeneous conditions such as NB, which has not been adequately explained using reductionist approaches, and is already benefitting from a more holistic and integrated approach to risk stratification. A systems model of NB offers benefits in terms of clinical management and therapeutic design for this disease, and could also assist developments in related areas of biology and pathology. Equally, NB is an idyllic condition to model due to extensive characterization with an array of quantitative biomarkers. NB, therefore, represents an opportunity for systems biology to prove its utility in making valid contributions to the biomedical sciences, not just with theoretical insights but with clinical applications to improve patient outcomes. 
Our in-house NB model revealed hypotheses that would be near impossible to conceive with a reductionist approach. If validated experimentally, these hypotheses may suggest the need for trials testing personalized NB MRD management strategies. Other research groups are also beginning to realise the value of modeling in NB [106-108], which will hopefully lead to further developments and investment in this approach.

It is neither necessary nor desirable to wait until all biomolecules are functionally characterized before utilizing models to study and improve the management of human disease. Hopefully this review has demonstrated that modeling can offer benefits in the current research climate, for example, by generating hypotheses and predictions that would not have been conceived using experimental methods alone. As measurement technologies continue to advance, mathematical modeling will become even more valuable in handling the ever-expanding wealth of data. A systems approach will increasingly offer benefit as a means of converting biological data into meaningful biological knowledge and viable therapeutic options.

\section{Expert commentary}

The last decade has seen remarkable collaborative efforts within the field of NB, with international cooperative groups working to gain knowledge of the disease by amassing large volumes of clinical and biological patient data. These efforts have recently paid dividends in the form of the INRG stratification, which is the most detailed risk-stratification system for NB to date. The clinical benefits of such a system should be to facilitate a more accurate calculation of risk in individual patients and, consequently, more appropriate assignment of treatment.

Risk stratification is likely to progress further in the near future, with the increasing availability of omics techniques allowing the routine global molecular analyses of biopsy specimens. The INRG cooperative has expressed hopes that in the future the majority of tumor samples will be characterized using omics technologies, and that the current risk stratification can be expanded further to incorporate the relevant biomarkers that arise as a result. Crucial to achieving these goals will be the continued development and evolution of computer algorithms for the automated processing and analysis of large datasets.

As in other cancer fields, therapeutic strategies in NB are beginning to expand beyond traditional cytotoxic therapy to incorporate more targeted biological therapies, with much investment into developing safe and effective alternative drugs. Retinoids represent the first biological therapy that has enjoyed clinical success in NB. These drugs exploit the biological properties of neuroblastic systems by switching their behavior from uncontrolled proliferation to differentiation and maturation. Retinoids thus manipulate the natural history of the disease towards involution and senescence.

Promising biological therapies for treatment of NB are even more abundant in the preclinical research setting. Some of these employ a guided-missile approach, exploiting NB-specific surface antigens, such as GD2, or NB-specific substrates, such as metaiodobenzylguanidine, in order to direct cytotoxic therapy to the site of the cancer cells. Alternative approaches target specific physiological processes that are relatively unique to cancer cells and are crucial for the development and propagation of the tumor,(e.g., inhibitors of angiogenesis or of the Trk receptor growth signaling pathway). These agents will begin to enter the clinical field in the near future and will hopefully present new opportunities for the management of high-risk disease.

\section{Five-year view}

In the next 5 years, the authors expect to see the increasing presence of systems biology both in biomedical research and in the clinical arena. Quantitative mathematical approaches will have increasing influence on our understanding of disease processes, and consequently on the development of new effective therapies. As systems biology success stories begin to emerge in specific areas, this will foster confidence in the approach and encourage the wider research community to adopt a more mathematical approach to biological systems. Furthermore, due to the emphasis on dynamic processes and regulation, systems-based discoveries in one research area will generate benefits for all related areas; for example, identification of common disease mechanisms in cancer biology.

In the field of NB, where there is already an established international research community, systems biology should particularly flourish. The collaborative efforts towards comprehensive molecular characterization will facilitate construction of mathematical models, which can be used to generate hypotheses and influence the nature of future measurements and data collection. In 5 years time, the authors hope to see marked evidence of a mutual exchange of ideas and hypotheses between the fields of modeling and simulation, and clinical research, allowing more rapid and exciting advances to be made in both areas.

With so many potential new therapies in the preclinical pipeline, the next 5 years is likely to witness further advances in biological therapies for NB. NB has various known biological behaviors and characteristics that could potentially be exploited for therapeutic gain, and significant effort is being directed towards achieving this clinically. The authors suggest that with contributions from systems biology, these goals could be realized more efficiently. The clinical potential of modeling and simulation is in predicting which extrinsic manipulations will produce the desired system response and, therefore, which compounds will produce the optimum clinical cure. Additionally, it could be feasible to predict which systems or system states will respond optimally to a particular perturbation and, therefore, which groups of individuals will derive most benefit from a given treatment. The next 5 years should clarify whether systems biology can deliver on these strategies of rational therapeutic design and personalized treatment planning, in order to improve clinical outcomes for individuals with NB.

\section{Financial \& competing interests disclosure}

The authors have no relevant affiliations or financial involvement with any organization or entity with a financial interest in or financial conflict with the subject matter or materials discussed in the manuscript. This includes employment, consultancies, honoraria, stock ownership or options, expert testimony, grants or patents received or pending, or royalties.

No writing assistance was utilized in the production of this manuscript. 


\section{Key issues}

- Neuroblastoma (NB) is a clinically heterogeneous condition, which has been described extensively in terms of prognostically valuable biomarkers. Multiple biomarkers can be combined to construct increasingly complex risk stratification systems (such as the recent International Neuroblastoma Risk Group), which guide clinical management.

- Despite advances in risk stratification and treatment planning, there are still subgroups of patients with NB facing a dismal prognosis. Further improvements in clinical outcome may be achieved through new approaches that make better use of existing biological data.

- Systems biology is a developing field that has potential value in NB research and clinical management. Systems biologists advocate a holistic approach to the study of biosystems, in which all system components carry equal importance. The emphasis of systems biology is on the dynamic interactions between individual molecules.

- Systems biology and NB biology can be viewed as mutually compatible and complementary fields. Systems biology work can facilitate explanation of the biological heterogeneity observed in NB and generate ideas for novel therapeutic options. Similarly, NB provides an ideal opportunity for systems biology to prove its utility in the clinical context, by virtue of its extensive molecular characterization.

- The practical execution of systems biology involves the use of mathematical modeling and simulation, that is the replication and manipulation of biosystems in a virtual framework. This process can generate novel hypotheses regarding biosystem characteristics and behaviors, and is complementary to laboratory-based experimental work.

\section{References}

Papers of special note have been highlighted as:

- of interest

-• of considerable interest

1 Maris JM, Hogarty MD, Bagatell R, Cohn SL. Neuroblastoma. Lancet 369(9579), 2106-2120 (2007).

- A good introduction to the clinical aspects of neuroblastoma (NB) presentation, assessment and management.

2 Castleberry RP. Neuroblastoma. Eur. J. Cancer 33(9), 1430-1437 (1997).

3 Grimmer MR, Weiss WA. Childhood tumors of the nervous system as disorders of normal development. Curr. Opin. Pediatr. 18(6), 634-638 (2006).

4 Brodeur GM. Neuroblastoma: biological insights into a clinical enigma. Nat. Rev. Cancer 3(3), 203-216 (2003).

- Some interesting insights into the biology and etiology of NB.

5 Tanaka T, Lehara T, Sugimoto T et al. Diversity in neuroblastomas and discrimination of the risk to progress. Cancer Lett. 228(1-2), 267-270 (2005).

6 D'Angio GJ, AE Evans, CE Koop. Special pattern of widespread neuroblastoma with a favourable prognosis. Lancet 1(7708), 1046-1049 (1971).

7 Monclair T, Brodeur GM, Ambros PF et al. The International Neuroblastoma Risk Group (INRG) staging system: an INRG Task Force report. J. Clin. Oncol. 27(2), 298-303 (2009).

8 Brodeur GM, Pritchard J, Berthold F et al. Revisions of the international criteria for neuroblastoma diagnosis, staging, and response to treatment. J. Clin. Oncol. 11(8), 1466-1477 (1993).

9 Shimada H, Chatten J, Newton WA Jr et al. Histopathologic prognostic factors in neuroblastic tumors: definition of subtypes of ganglioneuroblastoma and an agelinked classification of neuroblastomas. J. Natl Cancer Inst. 73(2), 405-416 (1984).

10 Shimada H, Ambros IM, Dehner LP, Hata J, Joshi W, Roald B. Terminology and morphologic criteria of neuroblastic tumors: recommendations by the International Neuroblastoma Pathology Committee. Cancer 86(2), 349-363 (1999).

11 London WB, Castleberry RP, Matthay KK et al. Evidence for an age cutoff greater than 365 days for neuroblastoma risk group stratification in the Children's Oncology Group. J. Clin. Oncol. 23(27), 6459-6465 (2005).

12 Riley RD, Heney D, Jones DR et al. A systematic review of molecular and biological tumor markers in neuroblastoma. Clin. Cancer Res. 10 (1 Pt 1), 4-12 (2004).

13 Cohn SL, Pearson AD, London WB et al. The International Neuroblastoma Risk Group (INRG) classification system: an INRG Task Force report. J. Clin. Oncol. 27(2), 289-297 (2009).

- Results from a large cohort study examining the prognostic value of various clinical and biological criteria in NB - an invaluable part of the NB evidence base.

14 Seeger RC, Brodeur GM, Sather H et al. Association of multiple copies of the N-myc oncogene with rapid progression of neuroblastomas. N. Engl. J. Med. 313(18), 1111-1116 (1985).

15 Bagatell R, Rumcheva P, London WB et al. Outcomes of children with intermediaterisk neuroblastoma after treatment stratified by MYCN status and tumor cell ploidy. J. Clin. Oncol. 23(34), 8819-8827 (2005).
16 Lehara T, Hosoi H, Akazawa $\mathrm{K}$ et al. $M Y C N$ gene amplification is a powerful prognostic factor even in infantile neuroblastoma detected by mass screening. Br. J. Cancer 94(10), 1510-1515 (2006).

17 Kaneko Y, Knudson AG. Mechanism and relevance of ploidy in neuroblastoma. Genes Chromosomes Cancer 29(2), 89-95 (2000).

18 Spitz R, Betts DR, Simon T et al. Favorable outcome of triploid neuroblastomas: a contribution to the special oncogenesis of neuroblastoma. Cancer Genet. Cytogenet. 167(1), 51-56 (2006).

19 Stallings RL, Carty P McArdle L et al. Molecular cytogenetic analysis of recurrent unbalanced $\mathrm{t}(11 ; 17)$ in neuroblastoma. Cancer Genet. Cytogenet. 154(1), 44-51 (2004).

20 Martinsson T, Sjöberg RM, Hedborg F, Kogner P. Deletion of chromosome 1p loci and microsatellite instability in neuroblastomas analyzed with shorttandem repeat polymorphisms. Cancer Res. 55(23), 5681-5686 (1995).

21 Bown N, Cotterill S, Lastowska M et al. Gain of chromosome arm 17q and adverse outcome in patients with neuroblastoma. N. Engl. J. Med. 340(25), 1954-1961 (1999).

22 Caren H, Abel F, Kogner P, Martinsson T. High incidence of DNA mutations and gene amplifications of the $A L K$ gene in advanced sporadic neuroblastoma tumours. Biochem. J. 416(2), 153-159 (2008).

23 Diskin SJ, Hou C, Glessner JT et al. Copy number variation at 1q21.1 associated with neuroblastoma. Nature 459(7249), 987-991 (2009).

24 Villamon E, Piqueras M, Mackintosh C et al. Comparison of different techniques for the detection of genetic risk-identifying 
chromosomal gains and losses in neuroblastoma. Virchows Arch. 453(1), 47-55 (2008).

25 Schramm A, Schulte JH, Astrahantseff K et al. Biological effects of TrkA and TrkB receptor signaling in neuroblastoma. Cancer Lett. 228(1-2), 143-153 (2005).

26 Taran K, Kobos J, Sitkiewicz A, Sporny S. Estimation of prognostic value of CD44 expression in neuroblastic tumours in children. Folia Neuropathol. 45(3), 126-132 (2007).

27 Castel V, Canete A. A comparison of current neuroblastoma chemotherapeutics. Expert Opin. Pharmacother. 5(1), 71-80 (2004).

28 Tonini GP, Pistoia V. Molecularly guided therapy of neuroblastoma: a review of different approaches. Curr. Pharm. Des. 12(18), 2303-2317 (2006).

29 Bruggeman FJ, Westerhoff HV. The nature of systems biology. Trends Microbiol. 15(1), 45-50 (2007).

30 Han JD. Understanding biological functions through molecular networks. Cell Res. 18(2), 224-237 (2008).

31 Kitano H. Systems biology: a brief overview. Science 295(5560), 1662-1664 (2002).

- A landmark paper for systems biology, providing a critical background.

32 Naylor S, Culbertson AW, Valentine SJ, Towards a systems level analysis of health and nutrition. Curr. Opin. Biotechnol. 19(2), 100-109 (2008).

33 Pache RA, Zanzoni A, Naval J, Mas JM, Aloy P. Towards a molecular characterisation of pathological pathways. FEBS Lett. 582(8), 1259-1265 (2008).

34 Ho RL, Lieu CA. Systems biology: an evolving approach in drug discovery and development. Drugs R D 9(4), 203-216 (2008).

35 Keskin O, Gursoy A, Ma B, Nussinov R. Towards drugs targeting multiple proteins in a systems biology approach. Curr. Top. Med. Chem. 7(10), 943-951 (2007).

36 Hopkins AL. Network pharmacology: the next paradigm in drug discovery. Nat. Chem. Biol. 4(11), 682-690 (2008).

37 Schrattenholz A, Soskic V. What does systems biology mean for drug development? Curr. Med. Chem. 15(15), 1520-1528 (2008).

38 Dietel M, Schafer R. Systems pathology - or how to solve the complex problem of predictive pathology. Virchows Arch. 453(4), 309-312 (2008).
39 Nicholson JK. Global systems biology, personalized medicine and molecular epidemiology. Mol. Syst. Biol 2, 52 (2006).

40 Feng X, Liu X, Luo Q, Liu BF. Mass spectrometry in systems biology: an overview. Mass Spectrom. Rev. 27(6), 635-660 (2008).

41 Teng X, Xiao H. Perspectives of DNA microarray and next-generation DNA sequencing technologies. Sci. China C Life Sci. 52(1), 7-16 (2009).

42 Klipp E, Liebermeister W. Mathematical modeling of intracellular signaling pathways. BMC Neurosci. 7(Suppl. 1), S10 (2006).

43 Watterson S, Marshall S, Ghazal P. Logic models of pathway biology. Drug Discov. Today 13(9-10), 447-456 (2008).

44 Hecker M, Lambeck S, Toepfer S, van Someren E, Guthke R. Gene regulatory network inference: data integration in dynamic models - a review. Biosystems 96(1), 86-103 (2009).

45 van Riel NA. Dynamic modelling and analysis of biochemical networks: mechanism-based models and model-based experiments. Brief Bioinform. 7(4), 364-374 (2006).

46 Ghosh D, Poisson LM. "Omics" data and levels of evidence for biomarker discovery. Genomics 93(1), 13-16 (2009).

47 Raffaghello L, Marimpietri D, Pagnan G et al. Anti-GD2 monoclonal antibody immunotherapy: a promising strategy in the prevention of neuroblastoma relapse. Cancer Lett. 197(1-2), 205-209 (2003).

48 Taggart D, Dubois S, Matthay KK. Radiolabeled metaiodobenzylguanidine for imaging and therapy of neuroblastoma. QJ Nucl. Med. Mol. Imaging 52(4), 403-418 (2008).

49 Brodeur GM. Genetics of embryonal tumours of childhood: retinoblastoma, Wilms' tumour and neuroblastoma. Cancer Surv. 25, 67-99 (1995).

50 Adhikary S, Eilers M. Transcriptional regulation and transformation by Myc proteins. Nat. Rev. Mol. Cell Biol. 6(8), 635-645 (2005).

51 Noble D. Computational models of the heart and their use in assessing the actions of drugs. J. Pharmacol. Sci. 107(2), 107-117 (2008).

52 Bizzarri M, Cucina A, Conti F, D’Anselmi F. Beyond the oncogene paradigm: understanding complexity in cancerogenesis. Acta Biotheoretica 56(3), 173-196 (2008).
53 Matthay KK, Villablanca JG, Seeger RC et al. Treatment of high-risk neuroblastoma with intensive chemotherapy, radiotherapy, autologous bone marrow transplantation, and 13-cis-retinoic acid. Children's Cancer Group. N. Engl. J. Med. 341(16), 1165 1173 (1999).

54 Reynolds CP, Matthay KK, Villablanca JG, Maurer BJ. Retinoid therapy of high-risk neuroblastoma. Cancer Lett. 197(1-2), 185-192 (2003).

55 Matthay KK, Reynolds CP, Seeger RC et al. Long-term results for children with high-risk neuroblastoma treated on a randomized trial of myeloablative therapy followed by 13-cis-retinoic acid: a children's oncology group study. J. Clin. Oncol. 27(7), 1007-1013 (2009).

56 Brodeur GM. Neuroblastoma: biological insights into a clinical enigma. Nat. Rev. Cancer 3(3), 203-216 (2003).

57 Hurlin PJ, Dezfouli S. Functions of myc:max in the control of cell proliferation and tumorigenesis. Int. Rev. Cytol. 238, 183-226 (2004).

58 Dang CV, O’Donnell KA, Zeller KI, Nguyen T, Osthus RC, Li F. The $c-M y c$ target gene network. Semin. Cancer Biol. 16(4), 253-264 (2006).

59 Alaminos M, Gerald WL, Cheung NK. Prognostic value of MYCN and ID2 overexpression in neuroblastoma. Pediatr. Blood Cancer 45(7), 909-915 (2005).

60 Hermeking H, Rago C, Schuhmacher M et al. Identification of CDK4 as a target of c-MYC. Proc. Natl Acad. Sci. USA 97(5), 2229-2234 (2000).

61 Yang W, Shen J, Wu M et al. Repression of transcription of the p27(Kip1) cyclindependent kinase inhibitor gene by c-Myc. Oncogene 20(14), 1688-1702 (2001).

62 Seoane J, Le HV, Massague J. Myc suppression of the p21(Cip1) Cdk inhibitor influences the outcome of the $\mathrm{p} 53$ response to DNA damage. Nature 419(6908), 729-734 (2002).

63 Staller P, Peukert K, Kiermaier A et al. Repression of $\mathrm{p} 15 \mathrm{INK} 4 \mathrm{~b}$ expression by Myc through association with Miz-1. Nat. Cell. Biol. 3(4), 392-399 (2001).

64 Galaktionov K, Chen X, Beach D. Cdc25 cell-cycle phosphatase as a target of c-myc. Nature 382(6591), 511-517 (1996).

65 Coller HA, Grandori C, Tamayo P et al. Expression analysis with oligonucleotide microarrays reveals that MYC regulates genes involved in growth, cell cycle, signaling, and adhesion. Proc. Natl Acad. Sci. USA 97(7), 3260-3265 (2000). 
66 Strieder V, Lutz W. E2F proteins regulate MYCN expression in neuroblastomas. J. Biol. Chem. 278(5), 2983-2989 (2003).

67 De los Santos M, Zambrano A, Aranda A. Combined effects of retinoic acid and histone deacetylase inhibitors on human neuroblastoma SH-SY5Y cells. Mol. Cancer Ther. 6(4), 1425-1432 (2007).

68 Cheung B, Hocker JE, Smith SA, Norris MD, Haber M, Marshall GM. Favorable prognostic significance of high-level retinoic acid receptor $\beta$ expression in neuroblastoma mediated by effects on cell cycle regulation. Oncogene 17(6), 751-759 (1998).

69 Villani MG, Appierto V, Cavadini E et al. 4-oxo-fenretinide, a recently identified fenretinide metabolite, induces marked G2-M cell cycle arrest and apoptosis in fenretinide-sensitive and fenretinideresistant cell lines. Cancer Res. 66(6), 3238-3247 (2006).

70 Yuza Y, Agawa M. Gene and protein expression profiling during differentiation of neuroblastoma cells triggered by 13-cis retinoic acid. J. Pediatr. Hematol. Oncol. 25(9), 715-720 (2003).

71 Reynolds CP, Wang Y, Melton LJ, Einhorn PA, Slamon DJ, Maurer BJ. Retinoic-acidresistant neuroblastoma cell lines show altered MYC regulation and high sensitivity to fenretinide. Med. Pediatr. Oncol. 35(6), 597-602 (2000).

72 Silchenko A, Tass P. Computational modeling of paroxysmal depolarization shifts in neurons induced by the glutamate release from astrocytes. Biol. Cybern. 98(1), 61-74 (2008).

73 Chan HS, Gaille BL, DeBoer G et al. $M Y C N$ protein expression as a predictor of neuroblastoma prognosis. Clin. Cancer Res. 3(10), 1699-1706 (1997).

74 Bordow SB, Norris MD, Haber PS, Marshall GM, Haber M. Prognostic significance of MYCN oncogene expression in childhood neuroblastoma. J. Clin. Oncol. 16(10), 3286-3294 (1998).

75 Cohn SL, London WB, Haung D et al. MYCN expression is not prognostic of adverse outcome in advanced-stage neuroblastoma with nonamplified MYCN. J. Clin. Oncol. 18(21), 3604-3613 (2000).

76 de Jong $\mathrm{H}$. Modeling and simulation of genetic regulatory systems: a literature review. J. Computat. Biol. 9(1), 67-103 (2002).

77 Ashyraliyev M, Fornekong-Nanfack Y, Kaandrop JA, Blom JG. Systems biology: parameter estimation for biochemical models. FEBS J. 276(4), 886-902 (2009).
78 Needham CJ, Bradford JR, Bulpitt AJ, Westhead DR. A primer on learning in Bayesian networks for computational biology. PLoS Comput. Biol. 3(8), e129 (2007).

79 Venancio TM, Aravind L. Reconstructing prokaryotic transcriptional regulatory networks: lessons from actinobacteria. J. Biol. 8(3), 29 (2009).

80 Herrgard MJ, Swainston N, Dobson P et al. A consensus yeast metabolic network reconstruction obtained from a community approach to systems biology. Nat. Biotech. 26(10), 1155-1160 (2008).

81 Ideker T, Thorsson V, Ranish JA et al. Integrated genomic and proteomic analyses of a systematically perturbed metabolic network. Science 292(5518), 929-934 (2001).

82 Chang WC, Li CW, Chen BS. Quantitative inference of dynamic regulatory pathways via microarray data. BMC Bioinformatics 6, 44 (2005).

83 Edgar D, Kenny S, Almond S, Murray P. Topography, stem cell behaviour, and organogenesis. Pediatr. Surg. Int. 20(10), 737-740 (2004).

84 Brandman O, Meyer T. Feedback loops shape cellular signals in space and time. Science 322 (5900), 390-395 (2008).

85 Ankers JM, Spiller DG, White MR, Harper CV. Spatio - temporal protein dynamics in single living cells. Curr. Opin. Biotechnol. 19(4), 375-380 (2008).

86 Werner S, Krieg T, Smola H. Keratinocyte - fibroblast interactions in wound healing. J. Invest. Dermatol. 127(5), 998-1008 (2007).

87 Fellin T. Communication between neurons and astrocytes: relevance to the modulation of synaptic and network activity. J. Neurochem. 108(3), 533-544 (2009).

88 Ruff RL. Neurophysiology of the neuromuscular junction: overview. Ann. NY Acad. Sci. 998, 1-10 (2003).

89 Sell H, Dietze-Schroeder D, Eckel J. The adipocyte-myocyte axis in insulin resistance. Trends Endocrinol. Metab. 17(10), 416-422 (2006).

90 Fernandez-Gonzalez R, Barcellos-Hoff MH, Ortiz-de-Solorzano C. A tool for the quantitative spatial analysis of complex cellular systems. IEEE Trans. Image Process. 14(9), 1300-1313 (2005).

91 Wu FT, Stefanini MO, MacGabhann F, Popel AS. A compartment model of VEGF distribution in humans in the presence of soluble VEGF receptor-1 acting as a ligand trap. PLoS ONE 4(4), e5108 (2009).
92 Lander ES, Linton LM, Birren B et al. Initial sequencing and analysis of the human genome. Nature 409(6822), 860-921 (2001).

93 International_HapMap_Consortium. The International HapMap Project. Nature 426(6968), 789-796 (2003).

94 Auer H, Newsom DL, Kornacker K. Expression profiling using Affymetrix GeneChip microarrays. Methods Mol. Biol. 509, 35-46 (2009).

95 Matsumura H, Krüger DH, Kahl G, Terauchi R. SuperSAGE: a modern platform for genome-wide quantitative transcript profiling. Curr. Pharm. Biotechnol. 9(5), 368-374 (2008).

96 Desiere F. Towards a systems biology understanding of human health: interplay between genotype, environment and nutrition. Biotechnol. Annu. Rev. 10, 51-84 (2004).

97 Mariman EC. Nutrigenomics and nutrigenetics: the 'omics' revolution in nutritional science. Biotechnol. Appl. Biochem. 44(Pt 3), 119-128 (2006).

98 Robles TF, Kiecolt-Glaser JK. The physiology of marriage: pathways to health. Physiol. Behav. 79(3), 409-416 (2003).

99 Varki A, Geschwind DH, Eichler EE. Explaining human uniqueness: genome interactions with environment, behaviour and culture. Nat. Rev. Genet. 9(10), 749-763 (2008).

100 Knutsson A. Health disorders of shift workers. Occup. Med. (Lond.) 53(2), 103-108 (2003).

101 Cole SW, Hawkley LC, Arevalo JM, Sung CY, Rose RM, Cacioppo JT. Social regulation of gene expression in human leukocytes. Genome Biol. 8(9), R189 (2007).

102 Brazma A, Hingamp P, Quackenbush J et al. Minimum information about a microarray experiment (MIAME) - toward standards for microarray data. Nat. Genet. 29(4), 365-371 (2001).

103 Bustin SA, Benes V, Garson JA et al. The MIQE guidelines: minimum information for publication of quantitative real-time PCR experiments. Clin. Chem. 55(4), 611-622 (2009).

104 Hucka M, Finney A, Bornsteiin BJ et al. Evolving a lingua franca and associated software infrastructure for computational systems biology: the Systems Biology Markup Language (SBML) project. Syst. Biol. (Stevenage) 1(1), 41-53 (2004).

105 Hunter PJ. The IUPS Physiome Project: a framework for computational physiology. 
Prog. Biophys. Mol. Biol. 85(2-3), 551-569 (2004).

106 Bilke S, Chen QR, Westerman F, Schwab M, Catchpoole D, Khan J. Inferring a tumor progression model for neuroblastoma from genomic data. J. Clin. Oncol. 23(29), 7322-7331 (2005).

107 Panetta JC, Schaiquevich P, Santana VM, Stewart CF. Using pharmacokinetic and pharmacodynamic modeling and simulation to evaluate importance of schedule in topotecan therapy for pediatric neuroblastoma. Clin. Cancer Res. 14(1), 318-325 (2008).

108 Hunsberger S, Albert PS, London WB. A finite mixture survival model to characterize risk groups of neuroblastoma. Stat. Med. 28(8), 1301-1314 (2009).

\section{Websites}

109 The National Centre for Text Mining, University of Manchester www.nactem.ac.uk/index.php

110 COPASI project: biochemical network simulator www.copasi.org
111 The Bioinformatics Research Centre, University of Glasgow www.brc.dcs.gla.ac.uk/projects/bps

\section{Affiliations}

- Jennifer A Logan Quantitative Molecular Medicine, Faculty of Medicine and Health Sciences, The Manchester Interdisciplinary Biocentre, University of Manchester, 131 Princess Street, Manchester, M1 7DN, UK; Now at the Medical Faculty, University of Liverpool, Liverpool L69 3BX, UK

- Martin E Kelly Quantitative Molecular Medicine, Faculty of Medicine and Health Sciences, The Manchester Interdisciplinary Biocentre, University of Manchester, 131 Princess Street, Manchester, M1 7DN, UK; Now at the Medical Faculty, University of Liverpool, Liverpool L69 3BX, UK

- Duncan Ayers Quantitative Molecular Medicine, Faculty of Medicine and Health Sciences, The Manchester Interdisciplinary Biocentre, University of Manchester, 131 Princess Street, Manchester, M1 7DN, UK
- Nicholas Shipillis

Doctoral Training Centre for Integrative Systems Biology, Manchester Interdisciplinary Biocentre, 131 Princess Street, Manchester, M1 7DN, UK.

\section{- Gerold Baier}

Doctoral Training Centre for Integrative Systems Biology, Manchester Interdisciplinary Biocentre, 131 Princess Street, Manchester, M1 7DN, UK.

- $\quad$ Philip JR Day

Quantitative Molecular Medicine, Faculty of Medicine and Health Sciences, The Manchester Interdisciplinary Biocentre, University of Manchester, 131 Princess Street, Manchester, M1 7DN, UK

Tel.: +441612751621

Fax: +44 1612751617

philip.j.day@manchester.ac.uk 